\title{
A Multi-Purpose Voice Controlled System on Android Platform
}

\author{
Shreya Dugar, Harsh Gupta \\ Dept. Of Computer Science and Engineering, Institute of Engineering \& Management, Kolkata, India
}

\begin{abstract}
This paper describes a method which helps in making Android operating system based mobile phone detect a voice command and perform the operation accordingly. We also introduce a new secured backup and restore approach for messages from the SMS logs, which helps to reduce the effort in saving and restoring them manually. Our approach is minimize time in efficient way for backup and restoring the data in the phone using android platform. We have used our own optimized and secured encryption and decryption technique to store and retrieve messages. It can also check the nearest ATM's, Hospitals etc, by sending and comparing the GPS location of the mobile phone to that stored in the database .
\end{abstract}

Keywords: GPS, Encryption and Decryption, SMS log, voice commands, data base.

\section{Introduction}

Android is an open-source Linux-based operating system designed mainly for smart phones and tablets. It is maintained as an open source project by Google. This open source code and licensing allows the developers and also the device manufacturers to modify the software according to their needs. Android is mainly based on ARM architecture platform

The paper helps to access an Android operating system based mobile phone easily. We can access a mobile phone even without touching it. The concept of voice signaling is utilized here by fetching data from the mobile. The data which could be fetched from the phone includes contacts, call logs, SMS logs, and current location of the mobile and can be used to call, message, use media features or locate a nearest place from the device's location, doing everything using voice commands. The user can access and perform operations through a Python platform. The application is made to run and controlled by voice commands (optional) in the Android phone.

We have used a concept to cache personal data of the user present in the phone by using an encryption technique in which we encode the original message received by the user from a number whose messages have to be kept personal using an algorithm and a randomly generated key in such a way that no one can even intercept what the message is, unless he knows the decryption algorithm and the key or the user id and password. These messages may contain important information which the user may not want others to read. We have proposed a technique by which the messages from a selected list of important numbers, will be encrypted and stored in the data base as soon as the message gets delivered in the phone. Whenever the user wants to retrieve the messages he can login to his id and decrypt and read them.

We have also incorporated the use of GPS in our application. GPS is a Global Positioning System based on satellite technology which provides location and time information in all weather conditions, anywhere on or near the Earth where there is an unobstructed line of sight to four or more GPS satellites. It helps to find the precise location of a person, or that of an asset to which it is attached and to record the position of the asset at regular intervals of time. This technique allows us to find the user's location. We use this GPS location of the device to locate the nearest ATM, Hospitals, Food Mart, Banks and few others nearest to user's location by using minimal path algorithm.

\section{Motivation}

The motivation behind this project was to make an application which could serve the people at large. There are people who do multi-tasking, for them an application as such will be helpful. For instance, those who are driving cars, they cant use their phones to make a call, so they can just open the application and make calls efficiently without putting their life at risk. Serving people who are new to a place and want to get to the nearest place (Hospitals, ATM's etc.) is also the motive behind making this application. Moreover GPS has been used because in our country we do not receive a very effective mobile data service so even when it is not available we can easily determine the nearest located place as and when required. Sometimes, we want to keep some messages confidential and those which we don't want others to access. So, we came up with an idea of encryption which will do the needful, thereby all the important messages are encoded and saved in a closed vault which nobody except the user with the pin code can access, hence giving full control over the privacy of the messages. 


\section{Android Application}

Android has a number of libraries to provide support for development of the android application. Android mobile application is responsible for executing stored commands. For developing this Android application, Android SDK and Python Idle were used. A comprehensive set of development tools is included in the Android software development kit (SDK).

\section{Contact}

In this paper, we are providing just an easy way for the users to contact someone from this app. This is all possible with the built-in APIs. The app can access user contacts that are stored on the phone by queryContent feature and stores it into newly created database and just by a voice command from user can make a call to required person either by his number or by name.

\section{Message}

By this developed application message can be sent via a voice command and can also retrieve unread messages in form of voice notes. There is a special facilities to save few numbers as per requirement as special numbers from which the messages received will be stored as encrypted message into the database and can be retrieved whenever required.

\section{Location}

The Android OS combined with a phone's GPS allows developers to access a user's location at any given moment within a distance. This location details of the user is used to obtain the nearest place as per the requirement of the user like atms, restaurants, malls etc,.

\section{Related Works}

The works related to the project are:

1. Blue-Droid: An Intelligent Library Management System on

Android Platform. IOSR Journal of Computer Engineering (IOSR-JCE)

e-ISSN: 2278-0661,p-ISSN: 2278-8727, Volume 16, Issue 4, Ver. V (Jul - Aug. 2014), PP 23-30

2. Android Based Appointment Scheduler and Location Helper

using file operation. IOSR Journal of Computer Engineering (IOSR-JCE)

e-ISSN: 2278-0661, p- ISSN: 2278-8727Volume 16, Issue 2, Ver. XI (Mar-Apr. 2014), PP 71-75

\section{Methodology}

Developing a system which incorporated the implementation of message storage in encrypted format on android platform with new encryption-decryption technique and provision for locating places like atms, hospitals etcetra nearby to the user just by usage of gps will be of much benefits to the user. We are mainly using the following encryption-decryption method:-

\section{Encryption Algorithm:}

Step 1: Find a random key which will help to encrypt the code.

Step 2: Add "' character to the end of the plain text till the length of the plain text is not completely divided by the length of the key.

Step 3: Start a loop which will run till all the characters of the plain text is not read one at a time.

Step 4: Determine the ASCII value of the character of key.

Step 5: Determine the ASCII value of the character of plain text.

Step 6: Add the ASCII value of the character and the key.

Step 7.1: Find the character value of the mod of the calculated sum and 128.

Step 7.2: If the length of ASCII value of the sum is greater than 128 then '@' after the character computed above, and add to the cipher text.

Step 7.3: Else add ' $\$$ ' after the characters computed above, and add it to the cipher text.

Step 8: Increase the value of the character of the key sequentially.

Step 9: If the value of the character reaches to its maximum plus 1 , then assign 0 to it. The flowchart for encrypting the messages is: 


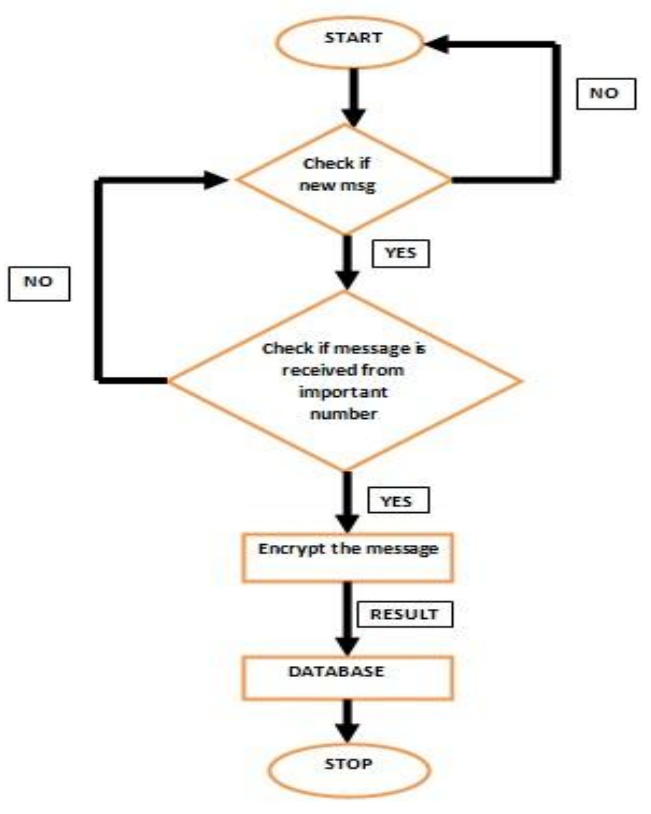

\section{Decryption Algorithm:}

Step 1: Check the every 2nd character of encrypted text whetehr its '@' or '\$'.

Step 2: If the character is "@" then determine the ASCII value of previous character and add 128 to it.

Step 3: Subtract the ASCII value of the presently pointed character of key from the above calculated value and then add it to the string after converting the above calculated value to its character.

Step 4: If the character is "\$" then determine the ASCII value of previous character and subtract the ASCII value of the presently pointed character of key from it.

Step 5: After that add it to the string after converting the above calculated value to its character.

Step 6: When the value of pointer determining index of key reaches the end it is again initialized back to 0 .

Step 7: Determine the index of " $"$ in the cipher text.

Step 8: Return the decrypted text with the length till the character before " "

The flowchart for decrypting the messages is:

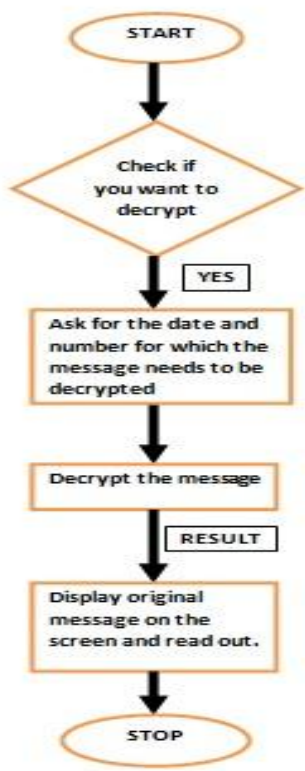

Now, for finding the nearby places different methodology is followed:

1. Initially present location of the user is detected. 
2. According to the requirement whether atms or malls etcetra data is retrieved from the database and minimum distance is calculated with respect to the user's location.

3. The name and address of place corresponding to that minimum distance is displayed.

The flowchart for calculating the minimum distance is:

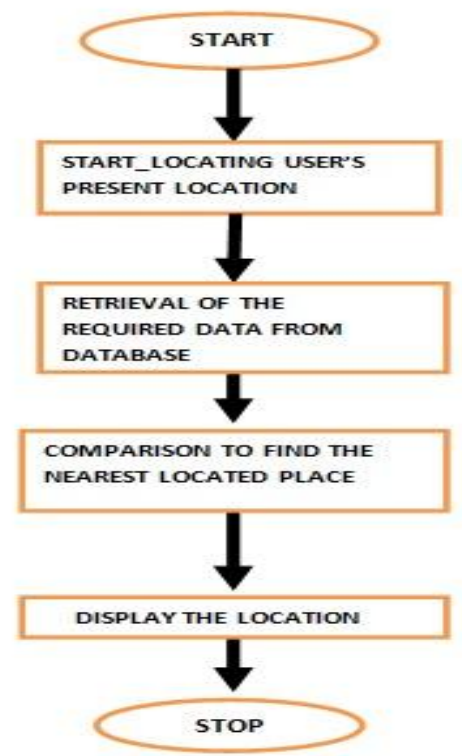

Algorithm for calculating the minimum distance:

Step 1: Determine the present location of user-the latitude and longitude using GPS.

Step 2: Connect to the database where the address and name s of places are stored.

Step 3: Choose what is to be located whether restaurant, malls or others.

Step 4: Start a loop to obtain all the chosen places in the database

Step 5: Subtract the latitude, longitude of every data in the database from user's present location and store in dlat and dlon respectively.

Step 6: Calculate $\mathrm{a}=(($ math.sin$($ dlat $/ 2)) *($ math.sin $($ dlat $/ 2))+$ math.cos(user's present latitude) $*$ math.cos(latitude of the currently compared place in database $) *(($ math.sin $($ dlon/2) $) *($ math.sin $($ dlon/2) $))$

Step 7: Calculate $x=($ math.tan(math.sqrt (a))+math.tan(math.sqrt (1-a)))/(1-(math.tan (math.sqrt (a))*math.tan (math.sqrt (1-a))))

Step 8: Store $\left(2 * a^{*} x\right)$ in c.

Step 9: Determine the distance in kms. by (radius of earth * c)

Step 10: if $d$ is found to be less than minimum value then

Step 11: Store the name and address of present location in a variable.

Step 12: End the loop

Step 13: Display the determined location which has minimum distance from the user.

\section{Software Requirments:}

Android Android is a mobile operating system (OS) based on the Linux kernel that is currently developed by Google. With a user interface based on direct manipulation, Android is designed primarily for touchscreen mobile devices such as smartphones and tablet computers. Android is popular with technology companies which require a ready-made, low-cost and customizable operating system for high-tech devices.

$>\quad$ SL4A I have used Scripting Layer for Android (SL4A) and python for developing my application. SL4A is a library that allows the creation and running of scripts written in various scripting languages directly on Android device.

Python Python is a high-level programming language which allows programmer to express concepts in fewer lines of code. Python is widely used as scripting language. The Java language is too heavy weight for some and is not entirely open source. Python has a wide usage and is open source. It also has seen the most interest as far as SL4A is concerned.

SQLite I have also used SQLite for my database related work. I use SQLite using sqlite3 module for python. I have used the different UI facades available in SL4A for user interactions. BLM is capable of 
performing different functions such as Searching any book, Requisition submission, Contact help, Book issue etc. An encryption algorithm is also included using ASCII conversion and some calculations. This encryption technique is used for enhancing the reliability of the software.

\section{Results:}

The following screenshots representing various working principles of the application are given below:

Whenever the voice command is to be received the following UI appears-

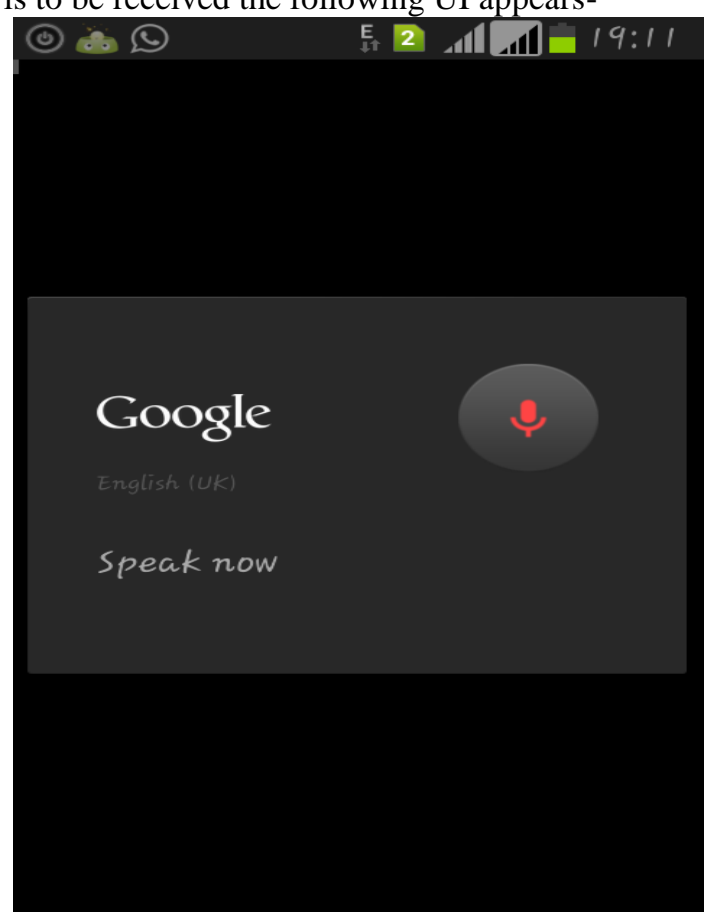

To show how the encryption decryption works:-

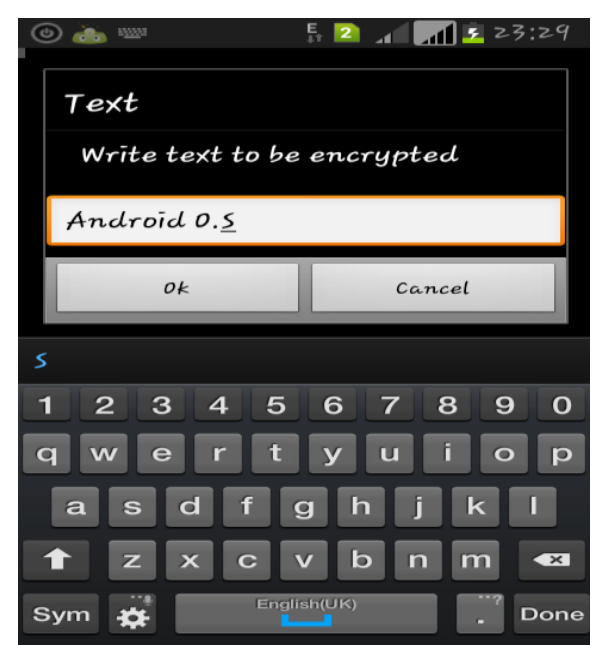



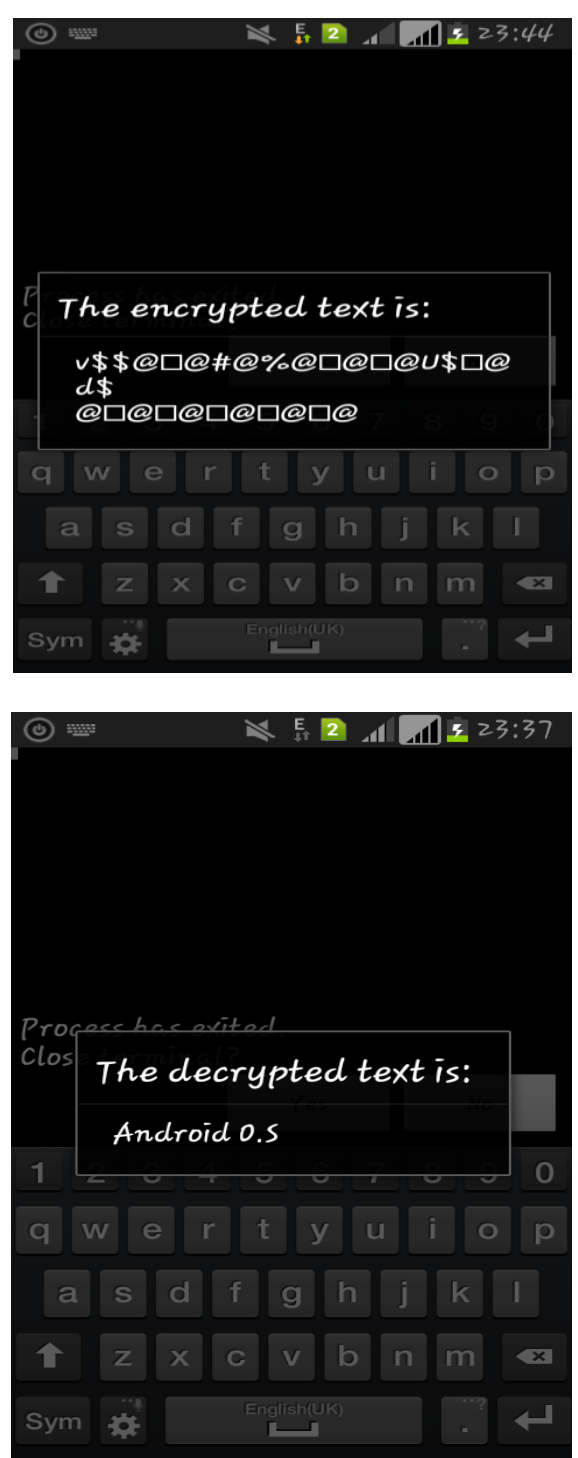

To show how the nearest hospital is is obtained when detected from a place in salt lake:-

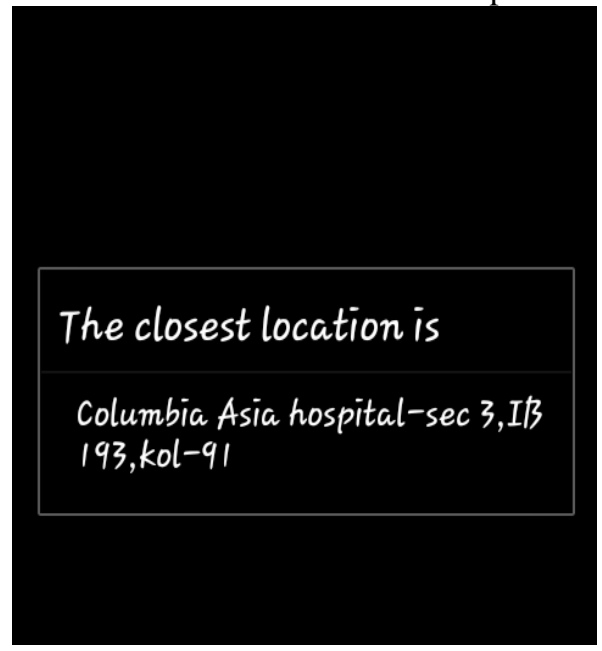


When nearest restaurant is found we obtain:

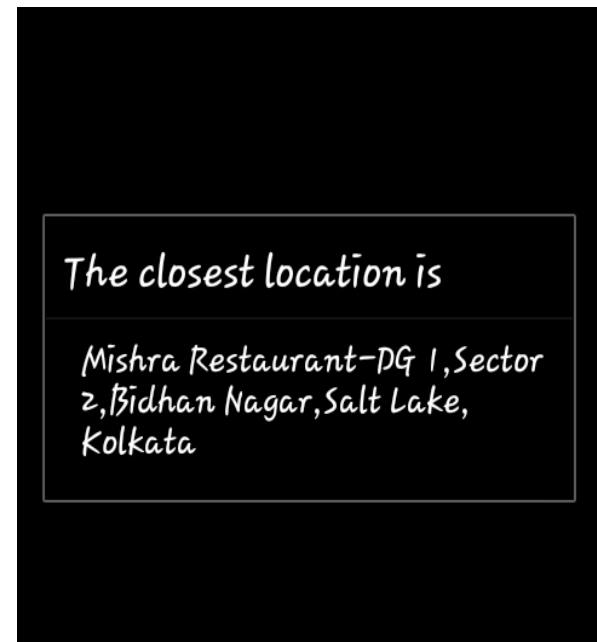

\section{Conclusion And Future Works}

From the various related works and the base paper, we have drawn a blueprint of the application. The proposed system has an application module framework of tracking GPS technology for effective information delivery and management. For location tracking we are using Geographical Positioning System. The raw data provided by GPS receiver is captured by the software and processed to extract the required location. The presented work here can be enriched with many refinements which are now in study phase. It however represents a highly encouraging approach towards a multiple tasks performing application with a new technique of encryption and decryption, new algorithm to store messages in an encrypted format and to perform various operations by voice commands. There is a lot of scope for making the application more secure by using voice processing as the security measure. Our proposed architecture offers a very promising solution for users who are in need of a secure and flexible access methodology using Android phone. 